Supporting Information to

\title{
Monolithic integration of silicon nanowire networks as a soft wafer for highly stretchable and transparent electronics
}

Taige Dong, Ying Sun, Zhimin Zhu, Xiaoxiang Wu, Junzhuan Wang, Yi Shi, Jun Xu, Kunji Chen and Linwei $\mathrm{Yu}^{*}$

${ }^{1}$ National Laboratory of Solid State Microstructures/School of Electronics Science and Engineering/Collaborative Innovation Center of Advanced Microstructures, Nanjing University, 210093 Nanjing, P. R. China, Corresponding email: yulinwei@nju.edu.cn

\section{Contents:}

Fig. S1: SEM images of catalyst formation and SiNW guided growth along step edges.

Fig. S2: Formation of double mini-steps along the edge lines, by alternative etching. 

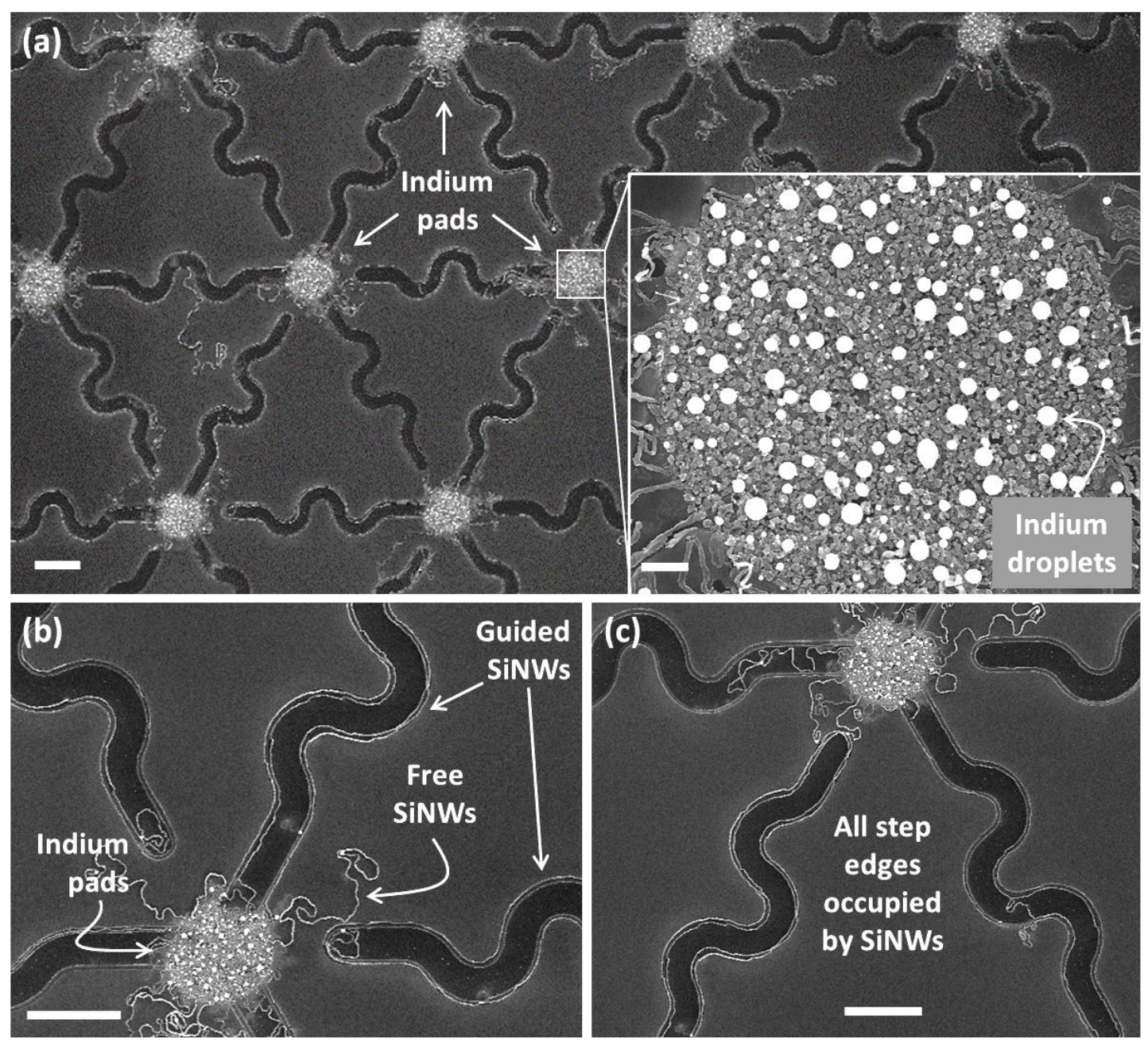

Figure S1. Catalyst formation and SiNW growth from indium (In) pads. a, SEM image of the hexagonal network layout with In pads located at the joints of guiding channels, with an inset showing the tiny In droplets left on the pads (after SiNW growth). b, $\mathbf{c}$ show the close SEM views of two In pads, where both guided or free SiNWs can grow, but only the former can travel over a long distance to connect the neighboring sites. Note that, basically all the guiding edges (with double-mini-steps) are occupied by two guided SiNWs. Scale bars in a-c stand for $10 \mu \mathrm{m}$, while that in the inset of a for $2 \mu m$. 

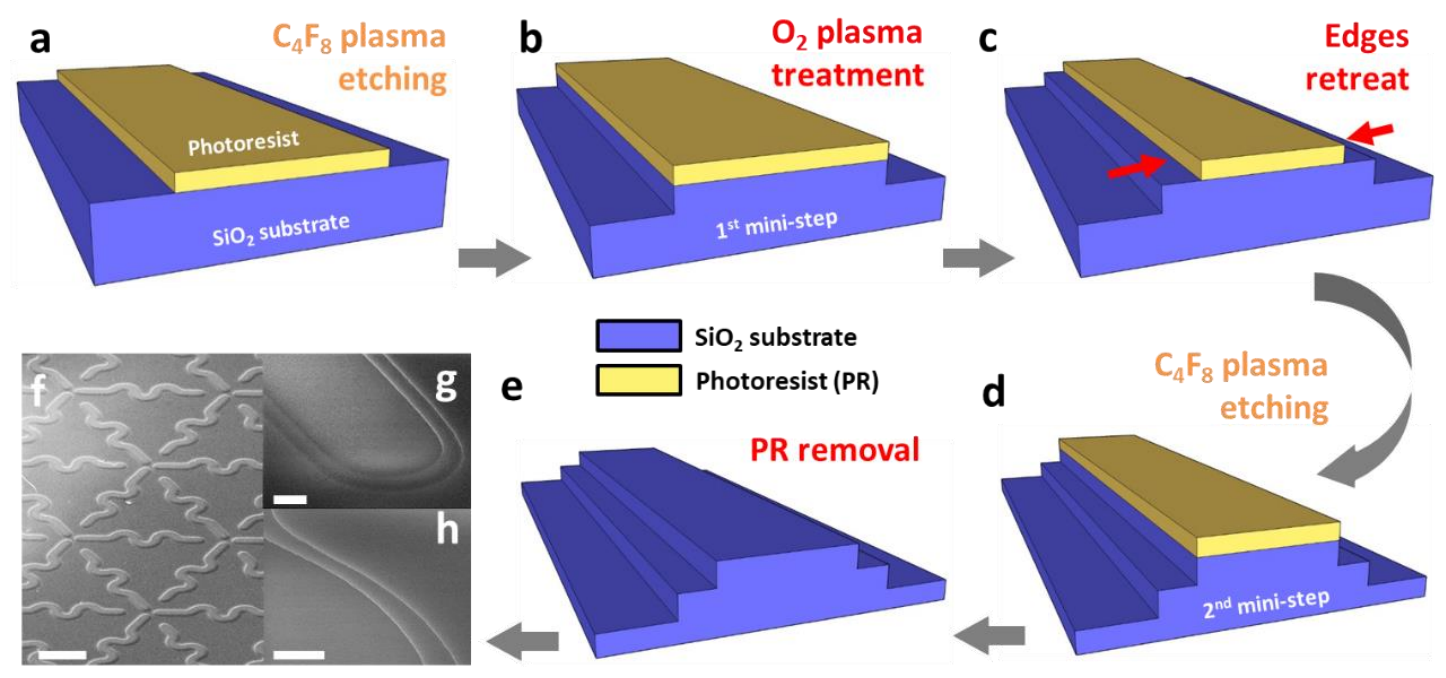

Figure S2. Illustration for the double mini-steps formation. a-e depict schematically the alternating $\mathrm{C}_{4} \mathrm{~F}_{8}-\mathrm{O}_{2}$ plasma etching process that leads to the formation of two mini-steps along a single guiding edge. f-h show the tilt-viewed SEM images of the double mini-steps guiding edges before catalyst pad deposition and SiNW growth. Scale bars in $\mathbf{f}, \mathbf{g}$ and $\mathbf{h}$ are $20 \mu m, 1 \mu m$ and $1 \mu m$, respectively. 